\title{
Influencia del paracetamol en la farmacocinética del cloramfenicol en pacientes con fiebre tifoídea
}

\author{
Q.F. María E. Bravo R. ${ }^{1}$; Dr. Jsidora Horwitz C. ${ }^{2}$; Q.F. Cecilia Contreras L: \\ Q.F. Irma Olya V.;Q.F. Aquiles Arancibia $\left.0.3^{3 *}\right)$ \\ Influence of paracetamol on chloramphenicol pharmacokinetics
}

\begin{abstract}
The effect of paracetamal on chloramphenicol (CAP) kinetics was studied in 12 children with typoid tever aged 3 to 15 years, recejving both drugs. A control group was formed by 9 children of the same age and disease, in which fever was controlled only by physical methods. Blood samples were obtained at differcnt time intervils after the first administration of CAP and at the steady state. No significant differences were found in the phamacokinetic parameters after the first CAP administration. At the stedy state C.AP blood concentrations were statistically different: in subjects receiving paracetamol they were lower than in controls $(\mathrm{p}<0.05$ ) Paracetamol ingestion is not indifferent for patients treated with clloramphenicol.
\end{abstract}

(Key words: paracetamol, chloramphenicol, pharmacokinetics, drug interactions).

El fármaco de elección en el tratamiento de las fiebres títicas es el cloramfenicol (CAF). Una de las formas comunes de administración en pediatria es la suspensión oral del ester palmitato de cloramfenicol (CAF-P). Un estudio anterior demostró que la biodisponibilidad del CAF.P en pacientes pediátricos es similar a la forma cristalina ${ }^{1}, y$ actualmente hay numerosas publicaciones en las que se informa del empleo de dicha forma farmacéutica en otras infecciones ${ }^{2-6}$. Se incluye frecuentemente en el tratamiento de la fiebre tifoídea un fármaco antipirético. Uno de los fármacos que se usa para estos fines es el paracetamol. Al igual que el CAF, el paracetamol se absorbe rápidamente desde el tracto gastrointestinal, es metabilizado por enzimas microsomales hepáticas y se excreta por vía renal después de su conjugación con ácidos glucurónico $(60 \%)$ y sulfúrico $(35 \%)$, principalmente 7 . Ambos fármacos se biotratnsforman en el hígado y a través del mismo sistema metabólico.

Diver şos autores han comunicado que el metabolismo del cloramfenicol es inhibido por otros fármacos $8 \cdot 10$. Por esto, nos ha parecido intere-

1. Instituto de Nutrición y Tecrología de los Alimentos, Universidad de Chite.

2. Departamento de Pediatría, División Ciencias Médicas Norte, Facultad de Medicina, Universidad de Chile, Hospital Rober to del Río.

3. Departamento de Ciencias Farmacológicas, Facultad de Cicncias Farmacéuticas.

(*) Trabajo financiado con fontos del DIB (M-678-8244). sante estudiar și los parámetros farmacocinéticos del CAF son influenciados por el paracetamol.

El objetivo de este trabajo es estudiar la influencia del paracetamol sobre los parámetros farmacocinéticos del cloramfenicol.

\section{MATERIAL Y METODOS}

\section{Pacientes:}

Se estudiaron 22 pacientés de ambos scxos que ingresaron al Hospital Roberto def Río entre los meses de Enero y Junio de 1984, con diagnóstico de ficbre entérica confirmada por examen bacteriológico de sangre, médula ósea, o ambas. El agente etiológico en todos los casos estudiados fue Salmonella typhi. En la selección de los pacientes se consideró yue no existiera tratamiento medicamentoso previo al diagnóstico y que el paciente no hubiera presentado la misma enfermedad anteriormente. Los pacientes se dividiet on en dos grupos:

Grupo A ( $=9$ ): Se les administró palmitato de cloramfenicol en dosis de $50 \mathrm{mg} \mathrm{x} \mathrm{kg}$ de peso día, fraccionado cada 12 horas. La edad de los pacientes fluctuó entre 5 y 12 años (promedio: $9,3 \pm 1,7$ años) y el peso entre 19,1 y 37,0 kg (promedio: $25,7 \pm 6,7 \mathrm{~kg}$ ). La fiebre en estos pacientes se trató por métodos físicos.

Grupo B $(n=12)$ : Se les administró palmitato de clocamfenicol en la misma dosis y forma que al Grupo A. La edad de los pacientes fluctuó entre 3 y 15 años (promedio $8,7 \pm 3,8$ años) y el peso entre 14,4 y $36,3 \mathrm{~kg}$ (promedio $24,8 \pm 8,2 \mathrm{~kg}$ ). La fiebre en estos pacientes se traxó administrando paracetamoi en dosis de $10 \mathrm{mg} \times \mathrm{kg}$ de peso cada 6 horas. Ambos fármacos se dieron por vía oral. Ia Tabla 1 muestra las características de los pacientes.

Preqio al inicio del tratamiento se tomó una muestra basal de $10 \mathrm{ml}$ de sangre, para realizar análisis de laboratorio (hematocrito, proteinas totales, albúmina, transaminasa glutámica pirúvica [GPT] transaminasa glutámica oxaloacética [GOT], uremia, nitrógeno ureico, 
Tabla 1.

Descripción de pacientes*

\begin{tabular}{|c|c|c|c|c|c|c|c|}
\hline \multirow{2}{*}{ Pacientes } & \multicolumn{2}{|c|}{ Sexo } & \multirow{2}{*}{$\begin{array}{l}\text { Edad } \\
\text { (años) }\end{array}$} & \multirow{2}{*}{$\begin{array}{l}\text { Peso } \\
\text { (kg) }\end{array}$} & \multirow{2}{*}{$\begin{array}{l}\text { Talta } \\
\text { (m) }\end{array}$} & \multirow{2}{*}{$\begin{array}{c}\text { Sup. } \\
\text { Corporal } \\
\left(m^{2}\right)\end{array}$} & \multirow{2}{*}{$\begin{array}{l}\text { Días de evolu- } \\
\text { ción previo al } \\
\text { tratamiento }\end{array}$} \\
\hline & $\mathrm{F}$ & $\mathbf{M}$ & & & & & \\
\hline $\begin{array}{l}\text { Grupo A } \\
(\mathrm{N}=9)\end{array}$ & 4 & 5 & $\begin{array}{c}9,3 \\
\pm 1,7\end{array}$ & $\begin{array}{l}25,7 \\
\pm 6,7\end{array}$ & $\begin{array}{c}1,31 \\
\pm 0,12\end{array}$ & $\begin{array}{c}0,99 \\
\pm 0,15\end{array}$ & $\begin{array}{l}13,9 \\
\pm 6,7\end{array}$ \\
\hline $\begin{array}{l}\text { Grupo B } \\
(\mathrm{N}=12)\end{array}$ & 6 & 6 & $\begin{array}{c}8,7 \\
\pm 3,8\end{array}$ & $\begin{array}{l}24,8 \\
\pm 8,2\end{array}$ & $\begin{array}{c}1,29 \\
\pm 0,20\end{array}$ & $\begin{array}{c}0,96 \\
\pm 0,23\end{array}$ & $\begin{array}{l}11,5 \\
\pm 3,3\end{array}$ \\
\hline
\end{tabular}

* Los resultados representan el promedio tla desviación estándar.

Tabla 2.

Resumen análisis đe faboratorio*

\begin{tabular}{|c|c|c|c|c|c|c|c|c|c|c|c|}
\hline Pacientex & $\begin{array}{l}\text { Hematoctito } \\
\text { (a)idl) }\end{array}$ & $\begin{array}{l}\text { Proluinas } \\
\text { Totales } \\
\text { ifidl) }\end{array}$ & $\begin{array}{l}\text { Albúmina } \\
\text { tu.k.j }\end{array}$ & $\begin{array}{l}\text { s:o' } \\
\text { (u.k.) }\end{array}$ & $\begin{array}{l}\text { (jp'T } \\
\text { (mgidl) }\end{array}$ & $\begin{array}{c}\text { BD } \\
\operatorname{teng} ; d \mid)\end{array}$ & $\begin{array}{c}\text { GT } \\
\text { (rigidl) }\end{array}$ & $\begin{array}{l}\text { [rres } \\
\text { (n)gidl) }\end{array}$ & $\begin{array}{l}\text { Nitróge- } \\
\text { no urajca } \\
\text { (mintidi) }\end{array}$ & $\begin{array}{l}\text { Crealinisa } \\
\text { suero } \\
\text { (mgidl) }\end{array}$ & $\begin{array}{c}\text { Cratinina } \\
\text { orina } \\
\text { (mgrdl) }\end{array}$ \\
\hline Grupo A & $\begin{array}{l}34,7 \\
\pm 3,2\end{array}$ & $\begin{array}{c}0,74 \\
\pm 0.64\end{array}$ & $\begin{array}{c}3,40 \\
\pm 0.47\end{array}$ & $\begin{array}{r}76.9 \\
\pm 11.7\end{array}$ & $\begin{array}{l}30, \hat{2} \\
\pm 4,4\end{array}$ & $\begin{array}{c}0,10 \\
\pm 0,066\end{array}$ & $\begin{array}{c}0,40 \\
\pm 0,14\end{array}$ & $\begin{array}{l}26.50 \\
\pm 3,25\end{array}$ & $\begin{array}{r}12.36 \\
\pm 1,59\end{array}$ & $\begin{array}{c}0,66 \\
\pm 0,15\end{array}$ & $\begin{array}{c}74,74 \\
\pm 17,11\end{array}$ \\
\hline Giтupo $B$ & $\begin{array}{r}-35.5 \\
\pm 4.4\end{array}$ & $\begin{array}{c}6.71 \\
\pm 0.67\end{array}$ & $\begin{array}{c}3,53 \\
\pm 0,60\end{array}$ & $\begin{array}{c}81,1 \\
\pm 11,6\end{array}$ & $\begin{array}{r}32,7 \\
\pm 4,5\end{array}$ & $\begin{array}{c}0,12 \\
\pm 0,04\end{array}$ & $\begin{array}{l}0,3 ? \\
0,10\end{array}$ & $\begin{array}{l}27,51 \\
\pm 7.84\end{array}$ & $\begin{array}{r}12,85 \\
\pm 3.65\end{array}$ & $\begin{array}{c}0,74 \\
\pm 0,17\end{array}$ & $\begin{array}{c}65,34 \\
\pm 12,81\end{array}$ \\
\hline
\end{tabular}

* Los rcaultados tepresentan el promedio \pm ja desyiación estrifidat.

bilitrubina directa, total y creatinina) y para determinar las ioncentraciones de fármaco a tiempo cero.

Los resultados de estos análisis se muestran en la Tabla 2.

Con cl abjeto de obtencr el perfil de concentración plasmatica de CAF y paracetamol versus ticmpo con una sola dosis, se tomó $1,0 \mathrm{ml}$ de sangre a los 60, 120,240, 360,480 y 600 minutos después de la administración de la primera dosis de fármacos. Para determinar las concentraciones de CAF y paracetamol en el estado dc equilibrio estable se tomaron muestras de sangre al terce dia de tratamiento: $1,0 \mathrm{ml}$ inmediatamente antes de la quinta dosis (predosis) a los 180 minutos, tiempo al cual se produce el pico maximo de concentración, y a los 720 minutos, es decir, inmediatamente antes de la dosis siguiente.

El CAF y el paracetamol se determinaron en plasmi por cromatografía líquida de alta resolución (HPLC) utilizando el método de Thies et al1] con ligeras modificaciones. Las determinaciones se realizaron en duplicado y se efectuaron controles de exactitud, especificidad, recuperabilidad y reproducibilidad.

\section{RESULTADOS}

En la figura 1A. muestra el perfil de concentraciones plasmáticas de ambos. grupos después de la primera dosis de cloramfenicol. Puede verse que la concentración plasmática đe CAF fue más baja a partir de las dos horas en pacientes

Figura 1: A) Concentración plasmática de cloramfenicol a través del tiempo despuós de la primera administración. Los valores representan el promedio \pm SEM. (Para propósitos de una buena figura los valores con paracetamol sq han corrido hacia la derecha B) Concentración plasmática del cloramfenicol en el estado estable (tercer día).

$0=$ Con paracetamol.

$\mathrm{X}=\mathrm{Sin}$ paracetamol. tratados con paracetamol, resultados que si bien no son significativos demuestran una tendencia. En la figura lB. se observa que al 3er. día de tratamiento las concentraciones de CAF se muestran significativamente más bajas en pacientes que recibieron paracetamol $(p<0,05)$.

Los parómetros farmacocinéticos obtenidos: constante de velocidad de eliminación de primer orden $K$, vida media $t 1 / 2$, constante de velocidad de absorción ka, vida media de absorción

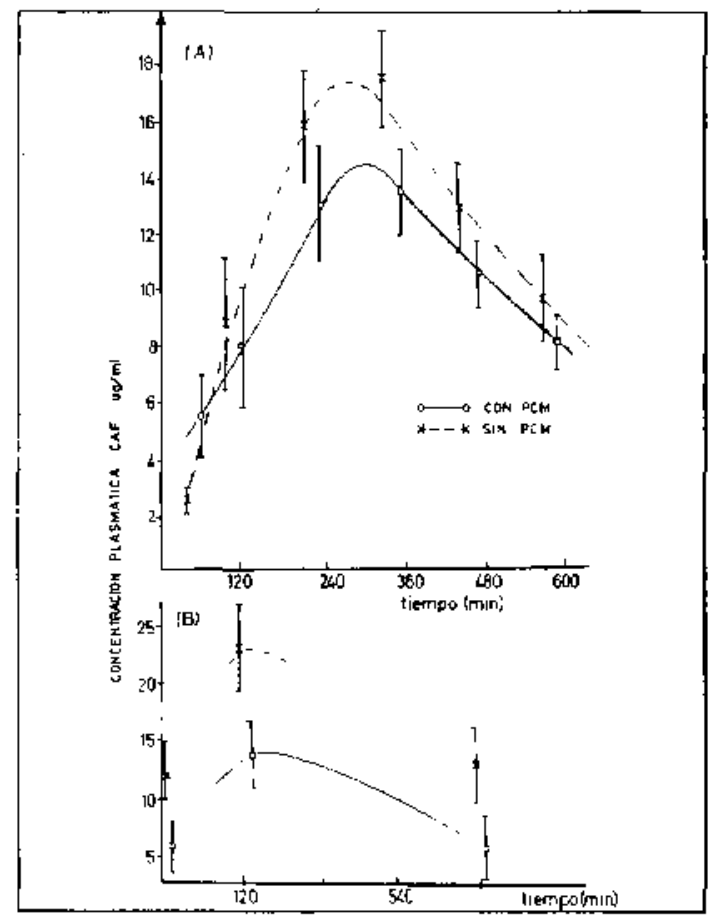


Tabla 3 :

Resumen de parámetros farmacocinétícos de $\mathrm{CAF}^{*}$

\begin{tabular}{|c|c|c|c|c|c|c|c|c|c|}
\hline Pacientes & ${ }^{\mathrm{K}}$ & $\begin{array}{c}\text { T } 1 / 2 \\
(\mathrm{~h}) \\
\end{array}$ & $\begin{array}{c}\mathrm{Ka} \\
\left(\mathrm{h}^{-1}\right)\end{array}$ & $\begin{array}{c}t / 1 \mathrm{ta} \\
\text { (h) } \\
\end{array}$ & $\begin{array}{l}\text { t pcak } \\
\text { (h) }\end{array}$ & $\begin{array}{l}\text { Cmáx. } \\
\text { (mg/l) }\end{array}$ & $\begin{array}{c}\mathrm{ABC} \\
\mathrm{mg} \cdot \mathrm{h} \\
1\end{array}$ & $\begin{array}{l}\mathrm{Cl} \\
\text { (j) }\end{array}$ & $\begin{array}{c}V d \\
(1 / \mathrm{kg})\end{array}$ \\
\hline $\begin{array}{l}\text { Grupo } A \\
(N=9)\end{array}$ & $\begin{array}{c}0,161 \\
\pm 0,077\end{array}$ & $\begin{array}{c}5,31 \\
\pm 2,62\end{array}$ & $\begin{array}{c}0,685 \\
\pm 0,403\end{array}$ & $\begin{array}{c}1,43 \\
\pm 0,91\end{array}$ & $\begin{array}{c}3,49 \\
\pm 1,93\end{array}$ & $\begin{array}{l}17,73 \\
\pm 5,20\end{array}$ & $\begin{array}{r}206,39 \\
\pm 85,46\end{array}$ & $\begin{array}{c}3,84 \\
\pm 1,91\end{array}$ & $\begin{array}{r}1,02 \\
\pm 0,35\end{array}$ \\
\hline $\begin{array}{l}\text { Crupo B } \\
(N=12)\end{array}$ & $\begin{array}{c}0,159 \\
\pm 0,058\end{array}$ & $\begin{array}{c}4,85 \\
\pm 1,58\end{array}$ & $\begin{array}{c}0,454 \\
\pm 0,199\end{array}$ & $\begin{array}{c}1,78 \\
\pm 0,74\end{array}$ & $\begin{array}{c}3,89 \\
\pm 1,27\end{array}$ & $\begin{array}{r}11,74 \\
\pm 6,83\end{array}$ & $\begin{array}{r}148,56 \\
\pm 60,37\end{array}$ & $\begin{array}{c}5,11 \\
\pm 2,27\end{array}$ & $\begin{array}{c}1,29 \\
\pm 0,82\end{array}$ \\
\hline
\end{tabular}

* Los resultados tepresentan el promedio † dusvación ustándar.

t 1/2 a, concentración máxima Cmáx, área bajo la curva de concentración plasmática versus tiempo $\mathrm{ABC}$, depuración, $\mathrm{Cl}$ y volumen de distribución aparente $\mathrm{Vd}$, para los pacientes de los Grupos A y B se muestran en la Tabla 3. Se observa que el $\mathrm{ABC}(\mathrm{mg} \times \mathrm{h} / \mathrm{l})$ y Cmáx ( $\mathrm{mg} \times \mathrm{l}$ ) son mayores en el Grupo $A$ que en el Grupo B, pero estas diferencias no son estadisticamente significativas $(p<0,05)$. En los otros parámetros farmacocinéticos no se aprecian diferencias entre ambos grupos de pacientes.

Tampoco hubo diferencias en los resultados clínicos entre ambos grupos de pacientes.

\section{DISCUSION}

Recienternente pudimos comprobar que la biodisponibilidad de CAF-P medida comparando las áreas bajo la curva de concentración plasmática versus tiempo, era similar a la de $C A F$-cristalino si bien el CAF.P demoraba más tiempo en alcanzar niveles plasmáticos adecuados.

En el presente estudio, la curva de concentra. ciones plasmáticas a través del tienpo (fig. 1) indica que si bien no hay diferencias significativas entre Jos dos grupos de pacientes después de la administración de la primera dosis de CAF, no es menos cierto que las concentraciones alcanzadas por el Grupo B (tratado con paracetamol) tienden a ser menores que las del Grupo A, tendencia que se observa más acentuadamente entre las 4 y 6 horas después de la administración del CAF.P y cuando los pacientes han recibido sólo una dosis de paracetamol. Estas diferencias en la concentración plasmática también se aprecian en el equilibrio estable que se alcanza luego de la administración de antibiótico durante tres dias (quinta dosis) como puede verse en la figura $1 \mathrm{~B}$.

En estos últimos años se ha liegado a establecer claramente que los efectos de muchos fármacos, cuando se administran simultáneamente con otro, no son necesariamente predecibles sobre la base del conocimiento de sus efectos cuando se administran solos. Esto ha llegado ha constituir un capítulo importante de la farmacologia cínica. Las interacciones entre fármacos pueden producirse por alteraciones farmacocinética a nivel de la absorción, transporte, metabolismo o excreción, o ambos ${ }^{12}$.

De nuestros resultados se puede concluir que las concentraciones más bajas de CAF encontradas al comienzo del tratamiento en el grupo con paracetamol pueden ser debidas a una variación en la absorción o a una disminución del volumen de distribución. Estos parámetros son ligeramente diferentes en ambos grupos pero no estadísticamente signifjcativos, en tanto que las cancentracjones significativamente más bajas en ese mismo grupo al momento del estado estable pueden ser debidas a competencia a nivel del metabolismo hepático en el denominado "complcjo citocromo P450"13. Sin embargo, no pueden sacarse de este trabajo conclusiones definitjvas, esto requería un estudio probablemente con animales de experimentación. Las concentraciones de paracetamol encontradas en los pacientes estudiados están en un rango entre 0,20-14,1 $\mathrm{ug} / \mathrm{l}$. Estas mediciones se efectuaron en todas las muesttas conteniendo CAF pero su determinación obedeció más a un criterio cualitativo que cuantitativo. Si bien las concentraciones de paracetamol son en algunos pacientes, subterapéuticas para su efecto antipirético, esto no invalida la posibilidađ de una interacción. La competencia por sustrato a nivel hepático pareciera posible porque esta requiere de un tiempo más largo y corresponde al hecho de que en nuestro estudio las concentraciones de CAF al tercer día de tratamiento son significativamente más bajas en los pacientes tratados con paracetamol (figura 1 B).

Las diferencias de concentración en el estado de equilibrio estable podrían deberse a diferencias en la absorción. Si bien las constantes de 
velocidad de absorción no son significativamente distintas, la del Grupo B es menor que Ja del Grupo A incidiendo en una menor cantidad de fármaco absorbida, que no se observa en un primer momento, ya que las $\mathrm{ABC}$ de ambos grupos son comparables, pero, después de varios días de tratamiento se puede ir acumulando este efecto para llegar a tener significancia.

No cabe ninguna duda, que el ideal de un buen estudio de un fărmaco que se administra por via oral requiere de un número alto de muestras de sangre que permitan definir las etapas de absorción y eliminación y en el dia en que se alcanza el estado estable. Sin embargo, en pacientes pediátricos resulta difícil seguir un protocolo que estabiezca un número aito de muestras; de ahí que algunos, autores como Done ${ }^{14}$ lo denominan "el huérfano terapéutico".

El presente estudio indica que no es indiferente el tratamiento con parecetamol en un paciente que está recibiendo CAF. Aún cuando los estudios en niños siempre presentan limitaciones impuestas por razones éticas y prácticas, se requerirían estudios más anplios para dilucidar la eventual interacción observada en este trabajo.

\section{RESUMEN}

Se estudió el efecto del paracetamol en la cinética del cloramfenicol en 12 niños con fiebres tificas (3-15 años) que recibieron ambos fárma. cos. El grupo control estuyo formado por 9 niños de la misma edad y la misma enfermedad en que la fiebre se controló por medios físicos. Se obtuvieron muestras de sangre a distintos tiempos después de la primera administración de CAF y al tercer día de ella, es decir cuando el fármaco está en su estado estable. Se encontró que no hubo diferencias significativas en los parámetros fartmacocinéticos después de la primera administración de CAF, en tanto que en el estado estable pudo observarse que las concentraciones plasmáticas fueron más bajas en los pacientes tratados con paracetamol, diferencias que resultaron esta. disticamente significativas $(p<0,05)$. Se concluye que no es indiferente el tratamiento con paracetamol en un paciente que está recibiendo cloramfenicol.

\section{REFERENCIAS}

1. Bravo, Moriz, E., Horwitz, I., Sinchez, A, y Arancibia Aquiles: Evaluación farmacocinética de dos formas orales de dosaje en niños con fiebres tíficas. Rev Chil Pedjatr 57: 121, 1986

2. Kauffman, R. et al: Relativa bioavailability of intravenous chloramphenicol succinate and oral chioramphenicol palmitate in infants and children. J Pediatr 99: $963,1981$.

3. Sack, M.M., Koup, J.R., Smith, A.L.: Chloramphenicol phasmacokinetics in infants and young children. Petiatrics 66: 579, 1980.

4. Tuomanen, E.I., Powell. K.R., Marks, M.I., Laferriere, C.I., Altemiller, D.H., Sack, C.. Smith, A.L.: Oral chloramphenicol in the treatment of hacmophilus influenzae meningitis. J Pediatr 99: 968,1981 .

5. Yogev, R., Kolling, W.M., William, T.: Pharmacokinetics comparison of intravenous and oral chloramphenicol in patientes with haemophilus influenzae meningitis. Pediatrics 67: 656, 1981 .

6. Sack. C., Koup. J., Ophein, E.K., Neely, N., Smith, $A$.: Chloramphenicol succinate kinetics in infants and young children. Pediatr Pharmacol 2: 93-103, 1982 .

7. Goodman and Giman: The pharmacological basis of therapeutics. 1th. Ed., New York, MacMillan Pub. Co., 1980.

8. Powell, D.: Bricf clinical and laboratory observations. Interaction among chloramphenicol, phenitoin and phenobarbital in a pediatric patient. J Pediatr 98: 1001, 1981.

9. Remmer, $H_{\text {.: }}$ The inhibition and stimulation of chloramphenicl conjugation by drugs. Post Med J 50 (Suppi 5): 28, 1974.

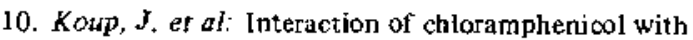
phenitoin and phenobarbital. Clin Pharm Therap 24: $572,1978$.

11. Thies, $R$, and Fischer, L.: High performance liquid chromatographic assay for chloramphenicol in biological fluids. Clin Chem 22: 778, 1978.

12. Drug Interactions. Ed. by P.L. Morselli, S. Garatini and S.N. Cohen.: Monographs of the Mario Negni. Institute for Pharmacological Research. Raven Press. New York, 1974.

13. Mersoner, C.H. and Smith, L.A.: The current status of chloramphenicol. Pediatrics 64: 348, 1979.

14. Done, A.K., Cohen, $S$ and Strebet, L.: Pcdiatric clinical pharmacologic and the "Therapeutic orphan". Ann Rev Phar, Tox 17: 561, 1977. 